Neurosurg Focus 12 (3):Article 2, 2002, Click here to return to Table of Contents

\title{
Weapons of mass destruction: biological
}

\author{
Ross R. Moquin, M.D., and Mary E. Moquin, R.N., M.S.N. \\ National Capital Consortium, Walter Reed Army Medical Center, Washington, D.C.; and Hospital \\ Education and Training, DeWitt Army Community Hospital, Fort Belvoir, Virginia
}

\begin{abstract}
Humans are susceptible to microbial infections from many sources. Biological warfare is the use of microbial forms of life to diminish the capabilities, disrupt the organization, and terrorize the noncombatant population of an adversary. This form of warfare has been used throughout history and has gained renewed interest with the current use of asymmetrical warfare. The civilized world has condemned its use by the implementation of treaties specifically against it. This is a brief review of some of the more easily used biological agents such as anthrax, plague, tularemia, Q fever, and smallpox. Each agent's biology, infectious route, and disease course will be discussed. Possible delivery systems and signs of outbreak will also be reviewed. There are few real neurosurgery-related implications in biological warfare. Neurosurgeons, as members and leaders of the healthcare community, must have the ability to recognize and initiate treatment when biological agents have been deployed. If there is widespread use of these inhumane agents, the neurosurgical community will not be able to practice the surgical art for which we have trained. New knowledge must be acquired so that we can best serve our patients and communities during times of extreme need.
\end{abstract}

\section{KEY WORDS • biological warfare • casualties • war • terrorism}

Biological warfare has been part of human conflict throughout the ages. Biological agents were used in many of the conflicts of the 20th century and their use is now reported daily in the headlines. Crude methods such as using dead or diseased animals to foul wells or gifts of contaminated blankets and clothes have been replaced by delivery systems of missiles, airplanes, and the postal service. Biological agents are gaining status as a terrorist's weapon of choice. ${ }^{2}$

The possibility that biological weapons will be used against us is no longer unthinkable. Until recently, healthcare practitioners have considered this topic suitable for only academic consideration. The importance of education regarding this subject cannot be overestimated. Before further terrorist actions occur or our soldiers engage against an aggressor likely to use biological weapons, healthcare providers need to be confident that they understand both the threat and appropriate medical countermeasures. ${ }^{4}$

There are few neurosurgery-related implications for biological warfare. Neurosurgeons, as members and leaders of the healthcare community, must be able to recognize

Abbreviations used in this paper: GI = gastrointestinal; US = United States; WMD = weapons of mass destruction. and initiate treatment when biological agents have been deployed. If there is widespread use of these inhumane agents, the neurosurgical community will not be able to practice. New knowledge must be acquired so that we can best serve our patients and communities during times of extreme need.

Defending against biological agents requires understanding of how an adversary might use them. ${ }^{4}$ Biological agents must be considered in terms of an evolving world, where advances in modern technology and weapons delivery systems (long-range cruise missiles with multiple warheads) have overcome some of their earlier physical limitations. An agent used in biological warfare need no longer be highly lethal to be effective, because to incapacitate and confuse a population on a widespread basis may actually cause greater disruption. Biological weapons may also be used in combination with other types of weapons, adding to the disruption produced by conventional weaponry. ${ }^{6}$ The method for delivery of biological warfare agents may be as simple and inconspicuous as attaching an off-the-shelf spray device to a car, truck, boat, or airplane that appears harmless to all who might observe the delivery vehicle. Current events suggest that nonconventional or terrorist use of biological agents is becoming more likely. 
Because initial symptoms caused by a biological warfare agent may be indistinguishable from those produced by endemic infections, a biological weapon may be capable of overtaking a military force or civilian population before the presence of the agent is even suspected. When one member of a unit or community falls victim, the disease may yet be silently present in others. ${ }^{6}$

The psychological and demoralizing impact of an infectious or toxic agent is likely to be more devastating than its physiological effect. Many biological agents, including bacteria, viruses, and toxins, can be used as weapons.

\section{REQUIREMENTS FOR AN IDEAL BIOLOGICAL WARFARE AGENT}

Although countless pathogens and toxins cause disease or intoxication in humans, few are as effective as biological weapons. For a number of reasons, tactics that are useful on a small scale, such as an assassination or a limited terrorist attack, are not as effective as WMD. There are several key factors that make biological pathogens or toxins suitable for use as WMD: availability or ease of production in sufficient quantity; the ability to cause either lethal or incapacitating effects in humans at doses that are achievable and deliverable; appropriate particle size in aerosol; ease of dissemination; stability (while maintaining virulence) after production, in storage, in weapons, and in the environment; and susceptibility of intended victims with nonsusceptibility of friendly forces. ${ }^{4}$ As discussed in the threat credibility article, the latter factor may not apply if the enemy has no concern for his own friendly forces.

Biological weapons are much more effective against unsuspecting, unprotected, and nonimmune civilian populations than against a fast-moving military organization. Biological weapons are well suited to the purpose of terrorist groups who seek such "soft" targets. Use of biological agents on a limited scale does not require great sophistication and can be accomplished with stealth. Largescale distribution of true WMD is more difficult.

\section{DETECTING BIOLOGICAL WARFARE AGENTS}

A satisfactory battlefield or community biological defense requires environmental monitoring systems that can detect the presence of toxic or infectious biological materials in the environment. Environmental biological detectors must be reliable, sensitive, and able to determine when a previously contaminated area is safe. If a biowarfare agent has been dispersed, it must be detected prior to its arrival over the target to allow time for personnel to don their protective equipment. Because of the delay in onset of symptoms and a limited number of detectors, the best detection is built by combining epidemiological and tactical intelligence strategies. In the civilian community, as on the battlefield, the level of suspicion should be appropriate to the threat level. New aerosol dispenser systems, the recent appearance of a chemical truck or storage tank, or breaks in a duct or water system may be the only indicator that a biological attack is in progress. In highthreat environments medical protection (vaccines and other prophylactic measures) may be indicated.
An epidemiological surveillance system must closely monitor unusual illnesses or outbreaks of disease. It is difficult for community or military medical advisors to know if an outbreak is consistent with a biowarfare attack unless background rates of disease for an area are known. Surveillance programs must be specifically tailored to the geographic area and focused on specific, diagnosable disease entities. Generic surveillance systems in which disease and injury are lumped into broad categories such as "respiratory" or "dermatological" are nearly useless as indicators of a biological warfare attack. Early and rapid analysis of specific epidemiological surveillance data may provide the first clue that such an attack has occurred.

\section{Anthrax (Bacillus anthracis)}

Anthrax (B. anthracis) appears to have a long history. The fifth and sixth plagues described in Exodus may have involved cutaneous anthrax in humans originating from domestic animals. Anthrax occurs worldwide and exists in the soil as a spore. It is not clear whether persistence in the soil results from multiplication of the organism in the soil or from cycles of bacterial amplification in infected animals whose carcasses then decay. Sporulation only occurs when the organism is exposed to air. Infection is incurred by direct contact, ingestion, or contact with infected creatures such as flies and vultures. Anthrax in humans is associated with agricultural, horticultural, or industrial exposure to infected animals or contaminated animal products. Direct contact with contaminated material leads to cutaneous disease, whereas ingestion of infected meat gives rise to oropharyngeal or GI forms of anthrax. Inhalation of a sufficient quantity of spores, which is usually observed only during generation of aerosols in an enclosed space associated with processing contaminated wool or hair, induces inhalational anthrax. The annual incidence of human-related anthrax has steadily declined from approximately 127 cases in the early 20 th century to approximately one per year for the past 10 years, and the majority of these cases have been cutaneous. Under natural conditions inhalational anthrax is exceedingly rare, with only 18 reported cases in the US in the 20th century. ${ }^{5}$

\section{Cutaneous Anthrax}

Over $95 \%$ of anthrax cases are cutaneous. The incubation period is 1 to 5 days. Cutaneous anthrax is in the differential diagnosis in cases in which there is a painless pruritic papule, vesicle, or ulcer, often with surrounding edema, that develops into an eschar. There may be local lymphadenitis and fever, but septicemia is very rare. Gram stain or culture of the lesion will usually confirm the diagnosis. When such cases are treated, the mortality rate should be less than $1 \%$.

\section{Oropharyngeal and GI Anthrax}

After an incubation period of 2 to 5 days, patients with oropharyngeal disease present with severe sore throat or a local oral/tonsillar ulcer, usually associated with fever, toxicity, and swelling of the neck due to cervical or submandibular lymphadenitis and edema. Dysphagia and respiratory distress may also be present. Initially, GI anthrax demonstrates nonspecific symptoms of nausea, vomiting, 
and fever, followed in most cases by severe abdominal pain. The presenting sign may be acute abdominal distress and may be associated with hematemesis, massive ascites, and diarrhea. Gastrointestinal anthrax is difficult to diagnose because it is rare and has nonspecific symptoms. The mortality rate in both forms may be as high as $50 \%$.

\section{Inhalational Anthrax}

After an incubation period of 1 to 6 days inhalational anthrax begins with nonspecific symptoms of malaise, fatigue, myalgia, fever, nonproductive cough, and mild chest discomfort. Symptoms usually persist for 2 to 3 days, and in some cases there may be a short period of symptomatic improvement. This period, however, is followed by the sudden onset of increasing respiratory distress with dyspnea, stridor, cyanosis, increased chest pain, diaphoresis, and edema of the chest and neck. Chest radiography usually demonstrates the characteristic widening of the mediastinum, frequently with pleural effusions. Pneumonia has not been a consistent finding. Meningitis is present in up to $50 \%$ of cases, and patients may present with seizures. Mortality rates are difficult to predict, but with treatment in early stages may be less than 20 to $30 \%$. The mortality rate in patients who are untreated after 4 to 5 days of symptoms develop may approach $100 \%$.

\section{Treatment and Precautions}

Ciprofloxacin is the drug of choice for anthrax prophylaxis and treatment. Cutaneous forms can be managed effectively with oral penicillin, although the intravenous form should be used in cases in which there is evidence of disseminated infection ( 2 million units intravenously every 6 hours), and gentamicin may be added. Tetracycline, erythromycin, and chloramphenicol have also been used successfully. Inhalational, oropharyngeal, and GI anthrax should be treated with 2 million units of intravenous penicillin and appropriate vasopressors, oxygen, and other supportive measures. Ciprofloxacin, doxycycline, and penicillin have been shown experimentally to be successful in avoiding death if initiated within a day after exposure to aerosolized anthrax. Optimum protection combines antibiotic agents with active immunization. Person-to-person transmission of anthrax has not been seen, but standard blood and body fluid precautions are recommended including gloves and impermeable gowns.

\section{Plague (Yersinia Pestis)}

Plague is a zoonotic infection caused by the Gram-negative $Y$. pestis. Plague occurs in pneumonic, bubonic, and septicemic forms. The three great human plague pandemics have caused a greater number of deaths than any other infectious agent in history. Plague is maintained in nature, predominantly in rodents, by a flea vector. $\mathrm{Hu}-$ mans are not necessary for persistence of the organism. Humans acquire the disease from animal fleas, contact with infected animals, or from other humans through aerosol or direct contact with infected secretions. In the most likely biological warfare scenario, plague would be spread through aerosol. A rapid person-to-person spread of fulminant pneumonia, characterized by blood-tinged sputum, would then ensue. If an enemy force were to release fleas infected with $Y$. pestis, soldiers would present with classic bubonic plague before a die-off in the local mammalian reservoir occurred. Bubonic plague is the most common naturally occurring form of the disease and is characterized symptomatically by painful lymphadenopathy and severe constitutional symptoms of fever, chills, and headache. Septicemic plague, without localized lymphadenopathy, occurs less commonly and is difficult to diagnose. Secondary pneumonia may follow either the bubonic or the septicemic form. Primary pneumonic plague is spread by airborne transmission. Diagnosis is established by isolating the organism from blood or other tissues. Rapid diagnosis may be established using fluorescent antibody stains of sputum or tissue specimens. Patients should be isolated and treated with aminoglycosides, preferably streptomycin, combined with chloramphenicol when meningitis is suspected or shock is present. The production of a licensed, killed, whole-cell vaccine to protect humans against the bubonic form has been discontinued. Prophylaxis is recommended using doxycycline or tetracycline in cases of asymptomatic contact or potential exposure. ${ }^{8}$ Plague is transmitted from person to person in droplets in the pneumonic form, and respiratory precautions are recommended until the patient has been treated for 3 full days. Otherwise, standard precautions are appropriate.

\section{Tularemia (Francisella tularensis)}

Tularemia is a zoonotic disease caused by infection with the Gram-negative, facultative intracellular bacterium $F$. tularensis. The organism is highly infectious via cutaneous and aerosol routes, but person-to-person transmission has not been seen. Infection follows contact with or ingestion of water contaminated by feces of infected rodents. A biological warfare attack in which aerosolized F. tularensis was used would probably produce pneumonia with or without accompanying mucous membrane lesions. The disease is characterized by fever, cough, localized skin or mucous membrane ulceration, regional lymphadenopathy, and occasional pneumonia. Patients with tularemia who do not receive appropriate antibiotic treatment may suffer a prolonged illness characterized by malaise, weakness, weight loss, and other symptoms that endure for months. A diagnosis is usually established by serological examination, because the organism is difficult to culture. Without antibiotic treatment, the mortality rate is 4 to $35 \%$. Treatment with streptomycin reduces the rate to between 1 and $2.5 \%$. Streptomycin is bactericidal, and patients treated usually respond within 48 hours of its administration. ${ }^{3}$ Other aminoglycosides such as gentamicin have been used with some success and are reasonable alternatives. Immediate postexposure prophylaxis with tetracycline prevents disease. A live, attenuated vaccine, available as an Investigational New Drug, is effective against aerosol infection. Laboratory personnel are required to use respiratory precautions, but person-to-person transmission is not seen. Standard precautions are appropriate.

\section{Q Fever (Coxiella Burnetii)}

A zoonotic disease caused by the rickettsia-like organism C. burnetii, $\mathrm{Q}$ fever is important to military medicine 
primarily because of its exceptional infectivity. ${ }^{1}$ The disease is transmitted mainly by inhalation of infected aerosols, and a single organism may cause infection in humans. The disease distribution is worldwide with livestock - goats, sheep, and cattle - as the primary mode. Contact with parturient animals or products of conception poses especially high risk, because the organism count is very high. The organism is very resistant to pressure and desiccation, and it may persist in a sporelike form in the environment for months after the source has left. Diagnosis of $\mathrm{Q}$ fever is made using serological testing. Treatment with tetracyclines is effective. Prevention is possible with a formalin-killed, whole-cell vaccine, but prior skin testing to exclude immune individuals is necessary to avoid severe local reactions to the vaccine. A Q fever vaccine is licensed in Australia but not in the US.

\section{Smallpox}

Despite the eradication of naturally occurring smallpox and the availability of a vaccine, the potential weaponization of variola virus continues to pose a threat militarily. This threat can be attributed to the aerosol infectivity of the virus, the relative ease of large-scale production, and an increasingly Orthopoxvirus-naive human populace. The incubation period for smallpox is 7 to 17 days. Smallpox is quickly diagnosed because of the synchronous eruption of the rash over the entire body, although very early stages of the rash can be mistaken for varicella. Symptoms progress to high fever, myalgia, abdominal pain, and delirium. Smallpox is highly contagious, and secondary spread constitutes a nosocomial hazard from the time at which enanthem occurs in an individual until the scabs have separated. Respiratory and body fluid isolation is critical. Treatment is supportive, although vaccinia vaccination, vaccinia immune globulin, and methisazone each possess some efficacy in postexposure treatment. All confirmed cases should be immediately reported to the Centers for Disease Control or the US Army Medical Research Institute of Infectious Diseases. Victims who sustain a secondary contact should be quarantined in respiratory isolation for 17 days after the exposure. Vaccinia vaccine is effective for preexposure prophylaxis against smallpox. ${ }^{7}$ Providers should perform clothing and surface decontamination after coming into contact with any infected patient.

\section{CONCLUSIONS}

Although the large-scale use of biological weapons to produce significant casualties on a strategic scale is both technically difficult and extraordinarily expensive, small- er-scale use by terrorists must be considered a serious threat. Biological precursors are relatively easy to obtain, and manufacturing techniques are not overly difficult. Simple delivery systems can be easily procured or manufactured. Most important, the psychological effects that may result from even a small-scale biological attack on an unprepared civilian population can be catastrophic. Although it is obvious that the threat is real, contingency planning must be based on a balance of available resources and the likelihood of attack.

\section{References}

1. Byrne WR: Q fever, in Sidell FR, Takafuji ET, Franz DR (eds): Medical Aspects of Chemical and Biological Warfare. Washington, DC: Office of the Surgeon General, 1997, pp 523-537

2. Eitzen EM, Takafuji ET: Historical overview of biological warfare, in Sidell FR, Takafuji ET, Franz DR (eds): Medical Aspects of Chemical and Biological Warfare. Washington, DC: Office of the Surgeon General, 1997, pp 415-424

3. Evans ME, Friedlander AM: Tularemia, in Sidell FR, Takafuji ET, Franz DR (eds): Medical Aspects of Chemical and Bological Warfare. Washington, DC: Office of the Surgeon General, 1997, pp 503-512

4. Franz DR, Parrott CD, Takafuji ET: The U.S. biological warfare and biological defense programs, in Sidell FR, Takafuji ET, Franz DR (eds): Medical Aspects of Chemical and Biological Warfare. Washington, DC: Office of the Surgeon General, 1997, pp 425-436

5. Friedlander AM: Anthrax, in Sidell FR, Takafuji ET, Franz DR (eds): Medical Aspects of Chemical and Biological Warfare. Washington, DC: Office of the Surgeon General, 1997, pp 467-478

6. Kortepeter M, Christopher G, Cieslak T, et al (eds): USAMRIID's Medical Management of Biological Casualties Handbook, ed 2. Frederick, MD U.S. Army Medical Research Institute of Infectious Diseases, 1996

7. McClain DJ: Smallpox, in Sidell FR, Takafuji ET, Franz DR (eds): Medical Aspects of Chemical and Biological Warfare. Washington, DC: Office of the Surgeon General, 1997, pp 539-559

8. McGovern TW, Friedlander AM: Plague, in Sidell FR, Takafuji ET, Franz DR (eds): Medical Aspects of Chemical and Biological Warfare. Washington, DC: Office of the Surgeon General, 1997, pp 479-502

Manuscript received January 18, 2002.

Accepted in final form February 12, 2002.

The opinions and views expressed in this article are those of the authors and do not reflect the official policy or position of the US Army, US Navy, Department of Defense, or US Government.

Address reprint requests to: Ross R. Moquin, M.D., Walter Reed Army Medical Center, 6900 Georgia Avenue, Building 2, Room 6442, Washington, D.C. 20307-5001. 